\title{
Pressure Transient Analysis of Dual Fractal Reservoir
}

\author{
Xiao-Hua Tan, ${ }^{1}$ Xiao-Ping Li, ${ }^{1}$ Jian-Yi Liu, ${ }^{1}$ Chuan Tang, ${ }^{1}$ and Jin-man Li $^{2}$ \\ ${ }^{1}$ State Key Laboratory of Oil and Gas Reservoir Geology and Exploitation, Southwest Petroleum University, \\ Chengdu 610500, China \\ ${ }^{2}$ Tianjin Exploration and Development Research Institute, CNOOC, Tanggu, Tianjin 300452, China
}

Correspondence should be addressed to Xiao-Hua Tan; xiaohua-tan@163.com

Received 21 May 2013; Revised 15 August 2013; Accepted 15 August 2013

Academic Editor: Bo Yu

Copyright (c) 2013 Xiao-Hua Tan et al. This is an open access article distributed under the Creative Commons Attribution License, which permits unrestricted use, distribution, and reproduction in any medium, provided the original work is properly cited.

A dual fractal reservoir transient flow model was created by embedding a fracture system simulated by a tree-shaped fractal network into a matrix system simulated by fractal porous media. The dimensionless bottom hole pressure model was created using the Laplace transform and Stehfest numerical inversion methods. According to the model's solution, the bilogarithmic type curves of the dual fractal reservoirs are illustrated, and the influence of different fractal factors on pressure transient responses is discussed. This semianalytical model provides a practical and reliable method for empirical applications.

\section{Introduction}

Numerous researchers have simulated the nonuniform distribution of fractures using fractal networks and have studied fluid flow behavior in fractured reservoirs. By assuming that the fracture network is fractal, Camacho-Velázquez et al. [1] studied the production decline behavior in a naturally fractured reservoir. Zhang and Tong [2] introduced a stresssensitive coefficient and built a transient pressure analysis model for fractal reservoirs which considers stress-sensitive effects.

Jafari and Babadagli [3] illustrated the 3D permeability distribution of a reservoir using outcrop, well log, and well test data which served as the basis for applying fractal networks to a reservoir. Zhang et al. [4] solved a nonlinear flow model for a stress-sensitive dual media fractal reservoir using a finite element method.

Previous scholars often embedded fractal structures into matrix networks by using straight or intersecting lines, but this did not correctly simulate well bottom radial flow. This type of radial flow has not been sufficiently studied in the underground seepage and oil development fields.

By referring to a plant lamina's bifurcation structure, Wechsatol et al. [5] used a tree-shaped fractal structure that connected center points to different circles. The fractal network could simulate the radial flow tending toward the well bottom. Based on their research, the construction method and optimization rules for tree-shaped fractal structures [6] were formulated.

$\mathrm{Xu}$ and $\mathrm{Yu}$ [7] presented a tree-shaped fractal flow model that considered the dynamic behavior of branching tubes in a tree-shaped fractal network. Based on this model, our model's transport properties and mass transfer capabilities $[8,9]$ were analyzed.

Given the capillary pressure effect, the starting pressure gradient influence, and pore fractal characteristics, Yun et al. [10] developed a fractal model that describes Bingham fluid flow in porous media. Based on Yun et al. [10], Wang et al. [11] proposed a tree-shaped fractal model that considered the influence of the starting pressure gradient on Bingham fluid seepage in a porous medium.

In this paper, fractures are simulated using a tree-shaped fractal network, as it accurately simulates radial flow tending to the well bottom, and the matrix system is simulated using fractal porous media. A transient flow model of dual fractal reservoirs is then presented by embedding the fracture network into a matrix system. Factors influencing the dynamic characteristics of transient pressure responses in dual fractal 


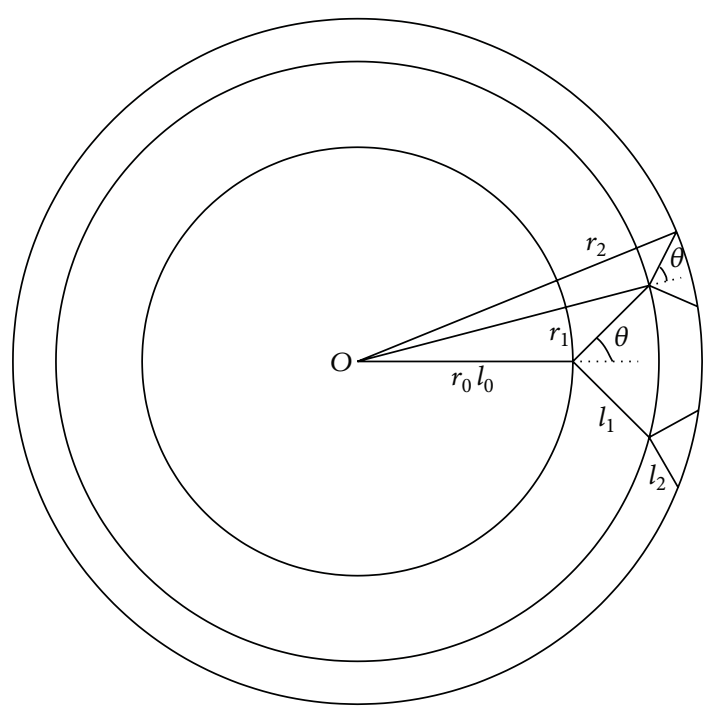

FIGURE 1: Dual fractal reservoir.

reservoirs are analyzed. This semianalytical model provides a practical and reliable method for empirical applications.

\section{Physical Model}

Figure 1 shows a well located in the reservoir center, $O$, with a thickness, $h$, and a well radius, $r_{w}$. The physical model assumptions are as follows.

(1) The fractal porous media is divided into $M$ annular sections in a tree-shaped fractal network.

(2) Each fracture section's properties are different, but the fluid properties are identical. Matrix permeability is much lower than that of the fracture.

(3) Rock and single-phase fluid are slightly compressible causing isothermal flow to be considered. Capillary pressure and gravity effects are neglected.

(4) Fluid flows to the wellbore only through the fracture system. Fluid flow in the matrix and the fracture system of each section satisfies the linear flow rule.

\section{Mathematical Model}

3.1. Matrix System. According to fractal geometry theory, the fractal scaling law can be used to describe the cumulative size distribution of pores in matrix system [12]:

$$
N_{m}(L \geq \lambda)=\left(\frac{\delta_{\max }}{\delta}\right)^{D_{f}}
$$

where $\delta$ is pore diameter, $\delta_{\max }$ is maximum pore diameter, and $D_{f}$ is the fractal dimension of the pore space.

Tortuosity is often used to describe flow path tortuosity, as flow in porous media is tortuous. The matrix flow path tortuosity is defined as [13]:

$$
T_{m}=\frac{L_{t}}{L_{0}}
$$

where $L_{t}$ is the actual length of the tortuous flow path and $L_{0}$ is the straight length along the macroscopic pressure gradient.

The matrix system porosity and permeability are defined as [14]:

$$
\begin{gathered}
\phi_{m}=\frac{\pi T_{m} D_{f}}{4\left(2-D_{f}\right)} \frac{\delta_{\max }^{2}}{A_{0}}\left[1-\left(\frac{\delta_{\min }}{\delta_{\max }}\right)^{2-D_{f}}\right], \\
K_{m}=\frac{\pi D_{f}}{128 T_{m}\left(4-D_{f}\right)} \frac{\delta_{\max }^{4}}{A_{0}}
\end{gathered}
$$

where $A_{0}$ is the unit cell area.

3.2. Fracture System. Fracture system parameters are directly generated from a tree-shaped fractal network. Sets of branch structures form the tree-shaped fractal network. During network generation, the branches on each level must end up on the same circle with all circles having the same center of origin, $O . N$ tubes which start at $O$ make up the tree-shaped fractal network. The tube's initial length and diameter are $l_{0}$ and $d_{0}$, respectively. The double branches $(n=2)$, whose angles are $\theta(\theta<\pi / 2)$ and total network branch levels are $M$, are applied in this network. Furthermore, two scale factors are used in this fractal network, length ratio, $\alpha$, and diameter ratio, $\beta$. The branch tube is assumed to be smooth, and tube wall thickness is ignored.

For the $k$ th level fracture, length is given by

$$
l_{k}=\alpha^{k} l_{0}
$$

For the $k$ th level fracture, diameter is

$$
d_{k}=\beta^{k} d_{0} \text {. }
$$

Distance from the well to each section's boundary is defined as the radial distance, which is expressed by [8]:

$$
r_{k}=\sum_{i=0}^{k} l_{i} \cos \theta=l_{0}\left[1+\frac{\alpha\left(1-\alpha^{k}\right) \cos \theta}{1-\alpha}\right] .
$$

Xu et al. [8] proposed the $k$ th level permeability expression of fracture system, which is expressed by

$$
K_{k}=\frac{d_{k}^{2}}{32} \frac{1}{T_{k}}
$$

The $k$ th section tortuosity of fracture systems can be obtained by the following expression:

$$
T_{k}=\frac{l_{k}}{r_{k}-r_{k-1}}= \begin{cases}1, & k=0 \\ \frac{1}{\cos \theta}, & k>0\end{cases}
$$

Substituting (6) and (9) into (8), the $k$ th section permeability in the fracture system is

$$
K_{f k}=N n^{k} \frac{d_{k}^{2}}{32} \frac{1}{T_{k}}= \begin{cases}\frac{N d_{0}^{2}}{32}, & k=0, \\ \frac{N n^{k} \beta^{2 k} d_{0}^{2} \cos \theta}{32}, & k>0 .\end{cases}
$$



by

For the $k$ th section, total system volume can be calculated

$$
V_{t k}= \begin{cases}\pi h r_{0}^{2}, & k=0 \\ \pi h\left(r_{k}^{2}-r_{k-1}^{2}\right), & k>0\end{cases}
$$

where $h$ is the reservoir thickness.

For the $k$ th section, pore volume of fracture systems can be calculated by

$$
V_{f t k}=N n^{k} \frac{\pi l_{k} d_{k}^{2}}{4}=\frac{N \pi n^{k} \alpha^{k} \beta^{2 k} l_{0} d_{0}^{2}}{4} .
$$

For the $k$ th section, total system volume, $V_{t k}$, is expressed as $V_{t k}=V_{m t k}+V_{f t k}$, where $V_{m t k}$ is total volume of the matrix system. $V_{m t k}$ is related to the pore volume of matrix system, $V_{m k}$, and expressed as $V_{m t k}=\phi_{m} V_{m k}$, where $\phi_{m}$ is the matrix system porosity. Thus, $V_{m k}$ can be calculated as

$$
V_{m k}=\phi_{m}\left(V_{t k}-V_{f t k}\right) \text {. }
$$

For the $k$ th section, porosity of a fracture system can be obtained by dividing (12) by (11):

$$
\phi_{f k}=\frac{V_{f t k}}{V_{t k}}= \begin{cases}\frac{N d_{0}^{2}}{4 h l_{0}}, & k=0, \\ \frac{N n^{k} \alpha^{k} \beta^{2 k} l_{0} d_{0}^{2}}{4 h\left(r_{k}^{2}-r_{k-1}^{2}\right)}, & k>0 .\end{cases}
$$

For the $k$ th section, porosity of a matrix system can be obtained by the following expression:

$$
\phi_{m k}=\frac{V_{m k}}{V_{t k}}=\phi_{m}\left(1-\phi_{f k}\right) .
$$

The permeability of a fracture system, $K_{f}$, and the porosity of a fracture system, $\phi_{f}$, do not change with the radial distance, $r$, in traditional double porosity (fracture and matrix system) reservoir transient flow models [15]. In order to compare dual fractal reservoir transient flow models with double porosity reservoir transient flow models, we have to clarify how to keep $K_{f}$ and $\phi_{f}$ independent of $r$ in dual fractal reservoir transient flow models.

Under the condition of double branches $(n=2)$, the permeability, $K_{f k}$, and porosity, $\phi_{f k}$, of every section in a fracture system are equal; that is,

$$
\begin{gathered}
K_{f k}=K_{f(k+1)}, \\
\phi_{f k}=\phi_{f(k+1)} .
\end{gathered}
$$

Substituting (10) and (14) into (16), we can obtain: $n=2$, $\alpha=1$, and $\beta=0.707$.

When $\beta$ is smaller than 0.707 , permeability of a fracture system increases with the radius of a dual fractal reservoir. When $\beta$ is larger than 0.707 , permeability of a fracture system decreases with the radius of a dual fractal reservoir.
3.3. Dual Fractal Reservoir. According to the physical model, the flow mathematical model of a dual fractal reservoir can be described as follows.

Governing differential equations in a dual fractal reservoir, we have the following.

For fracture system [15],

$$
\begin{array}{r}
\frac{K_{f k}}{\mu}\left(\frac{\partial^{2} p_{f k}}{\partial r^{2}}+\frac{1}{r} \frac{\partial p_{f k}}{\partial r}\right)+\frac{a K_{m}}{\mu}\left(p_{m k}-p_{f k}\right)=\frac{\varphi_{f k} C_{t f}}{3.6} \frac{\partial p_{f k}}{\partial t} \\
r_{k-1} \leq r \leq r_{k} .
\end{array}
$$

For matrix system [15],

$$
-\frac{a K_{m}}{\mu}\left(p_{m k}-p_{f k}\right)=\frac{\varphi_{m k} C_{t m}}{3.6} \frac{\partial p_{m k}}{\partial t} \quad r_{k-1} \leq r \leq r_{k} .
$$

Initial condition:

$$
p_{f k}(r, 0)=p_{m k}(r, 0)=p_{i} \quad(k=0,1, \ldots, M) .
$$

Interface connecting conditions of each zone, pressure continuity [16]:

$$
\left.p_{f k}\right|_{r=r_{k}}=\left.p_{f(k+1)}\right|_{r=r_{k}} \quad(k=0,1, \ldots, M-1) .
$$

Interface connecting conditions of each zone, rate continuity [16]:

$$
\left.\frac{\partial p_{f k}}{\partial r}\right|_{r=r_{k}}=\left.\frac{k_{f(k+1)}}{k_{f k}} \frac{\partial p_{f(k+1)}}{\partial r}\right|_{r=r_{k}} \quad(k=0,1, \ldots, M-1) .
$$

Well production condition [17]:

$$
q_{s f}=q+\frac{24 C}{B} \frac{d p_{w f}}{d t} \quad p_{w f}=\left.p_{f 1}\right|_{r=r_{w} e^{-s}}
$$

External boundary condition (infinite):

$$
p_{f M}(r \longrightarrow \infty, t)=p_{i} .
$$

External boundary condition (constant pressure):

$$
p_{f M}\left(r=r_{M}, t\right)=p_{i} .
$$

External boundary condition (closed):

$$
\left.\frac{\partial p_{f M}}{\partial r}\right|_{r=r_{M}}=0
$$

\section{Mathematical Model Solution}

To simplify the mathematical model and its solution, dimensionless parameters are defined as follows $[18,19]$.

The dimensionless pressure of the fracture system of the $k$ th section:

$$
p_{D f k}=\frac{K_{f k} h}{1.842 \times 10^{-3} q \mu B}\left(p_{i}-p_{f k}\right) .
$$


The dimensionless pressure of the matrix system of the $k$ th section:

$$
p_{\text {Dmk }}=\frac{K_{m k} h}{1.842 \times 10^{-3} q \mu B}\left(p_{i}-p_{m k}\right) .
$$

The dimensionless effective radius:

$$
r_{D e}=\frac{r}{r_{w}} e^{S}
$$

The dimensionless effective interface radius:

$$
r_{D e k}=\frac{r_{k}}{r_{w}} e^{S}
$$

The dimensionless effective time:

$$
t_{D e}=\frac{3.6 K_{f 0} t e^{2 S}}{\left(\varphi C_{t}\right)_{(f+m) 0} \mu r_{w}^{2}}
$$

The dimensionless effective wellbore storage coefficient:

$$
C_{D e}=\frac{C e^{2 S}}{2 \pi h\left(\phi C_{t}\right)_{(f+m) 0} r_{w}^{2}} .
$$

The fluid capacitance coefficient of the $k$ th section:

$$
\omega_{k}=\frac{\phi_{f k} C_{f t}}{\phi_{m k} C_{m t}+\phi_{f k} C_{f t}} .
$$

The interporosity flow coefficient of the $k$ th section:

$$
\lambda_{k}=a \frac{K_{m}}{K_{f k}} r_{w}^{2} \text {. }
$$

Substituting (26)-(33) into (17)-(25), dimensionless mathematical models are obtained [20].

For a fracture system, the governing differential equation in a dual fractal reservoir is

$$
\begin{gathered}
\frac{\partial^{2} p_{D f k}}{\partial r_{D e}^{2}}+\frac{1}{r_{D e}} \frac{\partial p_{D f k}}{\partial r_{D e}}+\lambda_{k} e^{-2 S}\left(\frac{K_{f k}}{K_{m}} p_{D m k}-p_{D f k}\right) \\
=\frac{\omega_{k}}{C_{D e}} \frac{K_{f 0}}{K_{f k}} \frac{\partial p_{D f k}}{\partial\left(t_{D e} / C_{D e}\right)} \quad r_{D e(k-1)} \leq r_{D e} \leq r_{D e k}
\end{gathered}
$$

For matrix system,

$$
\begin{array}{r}
-\lambda_{k} e^{-2 S}\left(p_{D m k}-\frac{K_{m}}{K_{f k}} p_{D f k}\right)=\frac{1-\omega_{k}}{C_{D e}} \frac{K_{f 0}}{K_{f k}} \frac{\partial p_{D m k}}{\partial\left(t_{D e} / C_{D e}\right)} \\
r_{D e(k-1)} \leq r_{D e} \leq r_{D e k}
\end{array}
$$

Initial condition:

$$
p_{D f k}\left(r_{D e}, 0\right)=p_{D m k}\left(r_{D e}, 0\right)=0 \quad(k=0,1, \ldots, M) .
$$

Interface connecting conditions of each zone, pressure continuity:

$$
\left.p_{D f k}\right|_{r_{D e}=r_{D e k}}=\left.p_{D f(k+1)}\right|_{r_{D e}=r_{D e k}} \quad(k=0,1, \ldots, M-1) .
$$

Interface connecting conditions of each zone, rate continuity:

$$
\begin{array}{r}
\left.\frac{\partial p_{D f k}}{\partial r_{D}}\right|_{r_{D e}=r_{D e k}}=\left.\frac{K_{f(k+1)}}{K_{f k}} \frac{\partial p_{D f(k+1)}}{\partial r_{D e}}\right|_{r_{D e}=r_{D e k}} \\
(k=0,1, \ldots, M-1) .
\end{array}
$$

Well production condition:

$$
\frac{d p_{w D}}{d\left(t_{D e} / C_{D e}\right)}-\left.\left(r_{D e} \frac{\partial p_{D f 0}}{\partial r_{D e}}\right)\right|_{r_{D e}=1}=1 \quad p_{w D}=\left.p_{D f 0}\right|_{r_{D e}=1}
$$

External boundary condition (infinite):

$$
p_{D f M}\left(r_{D e} \longrightarrow \infty, t_{D e}\right)=0 \text {. }
$$

External boundary condition (constant pressure):

$$
p_{D f M}\left(r_{D e}=r_{D e M}, t_{D e}\right)=0 \text {. }
$$

External boundary condition (closed):

$$
\left.\frac{\partial p_{D f M}}{\partial r_{D e}}\right|_{r_{D e}=r_{D e M}}=0 .
$$

The flow mathematical model in Laplace space is obtained by taking the Laplace transformation of (34)-(42) based on $t_{D e} / C_{D e}$. The flow mathematical model is as follows [20].

For fracture system,

$$
\begin{gathered}
\frac{d^{2} \bar{p}_{D f k}}{d r_{D e}^{2}}+\frac{1}{r_{D e}} \frac{d \bar{p}_{D f k}}{d r_{D e}}+\lambda_{k} e^{-2 S}\left(\frac{K_{f k}}{K_{m}} \bar{p}_{D m k}-\bar{p}_{D f k}\right) \\
=\frac{\omega_{k}}{C_{D e}} \frac{K_{f 0}}{K_{f k}} z \bar{p}_{D f k} \quad r_{D e(k-1)} \leq r_{D e} \leq r_{D e k} .
\end{gathered}
$$

For matrix system,

$$
\begin{aligned}
& -\lambda_{k} e^{-2 S}\left(\bar{p}_{D m k}-\frac{K_{m}}{K_{f k}} \bar{p}_{D f k}\right)=\frac{1-\omega_{k}}{C_{D e}} \frac{K_{f 0}}{K_{f k}} z \bar{p}_{D m k} \\
& r_{D e(k-1)} \leq r_{D e} \leq r_{D e k} .
\end{aligned}
$$

Initial condition:

$$
\begin{array}{r}
\bar{p}_{D f k}\left(r_{D e}, 0\right)=\bar{p}_{D m k}\left(r_{D e}, 0\right)=0 \\
(k=0,1, \ldots, M) .
\end{array}
$$

Interface connecting conditions of each zone, pressure continuity:

$$
\left.\bar{p}_{D f k}\right|_{r_{D e}=r_{D e k}}=\left.\bar{p}_{D f(k+1)}\right|_{r_{D e}=r_{D e k}} \quad(k=0,1, \ldots, M-1) .
$$

Interface connecting conditions of each zone, rate continuity:

$$
\begin{array}{r}
\left.\frac{d \bar{p}_{D f k}}{d r_{D e}}\right|_{r_{D e}=r_{D e k}}=\left.\frac{K_{f(k+1)}}{K_{f k}} \frac{d \bar{p}_{D f(k+1)}}{d r_{D e}}\right|_{r_{D e}=r_{D e k}} \\
(k=0,1, \ldots, M-1) .
\end{array}
$$


Well production condition:

$z \bar{p}_{w D}-\left.\left(r_{D e} \frac{d \bar{p}_{D f 0}}{d r_{D e}}\right)\right|_{r_{D e}=1}=\frac{1}{z} \quad \bar{p}_{w D}=\left.\bar{p}_{D f 0}\right|_{r_{D e}=1}$.

External boundary condition (infinite):

$$
\bar{p}_{D f M}\left(r_{D e} \longrightarrow \infty, z\right)=0 .
$$

External boundary condition (constant pressure),

$$
\bar{p}_{D f M}\left(r_{D e}=r_{D e M}, z\right)=0 .
$$

External boundary condition (closed).

$$
\left.\frac{\partial \bar{p}_{D f M}}{\partial r_{D e}}\right|_{r_{D e}=r_{D e M}}=0 .
$$
by

In (43) and (44), the general solution of $\bar{p}_{D f k}$ is calculated

$$
\begin{array}{r}
\bar{p}_{D f k}=A_{k} I_{0}\left(r_{D e k} \sqrt{S_{k}(z)}\right)+B_{k} K_{0}\left(r_{D e k} \sqrt{S_{k}(z)}\right) \\
(k=0,1, \ldots, M) .
\end{array}
$$

The derivative of $\bar{p}_{D f k}$ in (52) is calculated by

$$
\begin{aligned}
\frac{d \bar{p}_{D f k}}{d r_{D e}}= & \sqrt{S_{k}(z)} A_{k} I_{1}\left(r_{D e k} \sqrt{S_{k}(z)}\right) \\
& -\sqrt{S_{k}(z)} B_{k} K_{1}\left(r_{D e k} \sqrt{S_{k}(z)}\right) \quad(k=0,1, \ldots, M) .
\end{aligned}
$$

Substitute (52) and (53) into well production condition equation (48);

$$
\begin{gathered}
z \bar{p}_{w f D}-\sqrt{S_{0}(z)} A_{0} I_{1}\left(\sqrt{S_{0}(z)}\right) \\
+\sqrt{S_{0}(z)} B_{0} K_{1}\left(\sqrt{S_{0}(z)}\right)=\frac{1}{z}, \\
\bar{p}_{w f D}=A_{0} I_{0}\left(\sqrt{S_{0}(z)}\right)+B_{0} K_{0}\left(\sqrt{S_{0}(z)}\right) .
\end{gathered}
$$

Substitute (52) and (53) into interface connecting condition equation (46);

$$
\begin{aligned}
A_{k} I_{0} & \left(r_{D e k} \sqrt{S_{k}(z)}\right)+B_{k} K_{0}\left(r_{D e k} \sqrt{S_{k}(z)}\right) \\
= & A_{k+1} I_{0}\left(r_{D e k} \sqrt{S_{k+1}(z)}\right) \\
& +B_{k+1} K_{0}\left(r_{D e k} \sqrt{S_{k+1}(z)}\right) \quad(k=0,1, \ldots, M-1) .
\end{aligned}
$$

Substitute (52) and (53) into interface connecting condition equation (47);

$$
\begin{gathered}
A_{k} \sqrt{S_{k}(z)} I_{1}\left(r_{D e k} \sqrt{S_{k}(z)}\right)-B_{k} \sqrt{S_{k}(z)} K_{1}\left(r_{D e k} \sqrt{S_{k}(z)}\right) \\
=\frac{K_{f(k+1)}}{K_{f k}} A_{k+1} \sqrt{S_{k}(z)} I_{1}\left(r_{D e k} \sqrt{S_{k}(z)}\right) \\
-\frac{K_{f(k+1)}}{K_{f k}} B_{k+1} \sqrt{S_{k}(z)} K_{1}\left(r_{D e k} \sqrt{S_{k}(z)}\right) \\
(k=0,1, \ldots, M-1) .
\end{gathered}
$$

Substitute (52) and (53) into external boundary condition equations (49)-(51);

$$
A_{M}=0
$$

$$
\begin{aligned}
& A_{M} I_{0}\left(r_{D e M} \sqrt{S_{M}(z)}\right)+B_{M} K_{0}\left(r_{D e M} \sqrt{S_{M}(z)}\right)=0, \\
& A_{M} I_{1}\left(r_{D e M} \sqrt{S_{M}(z)}\right)-B_{M} K_{1}\left(r_{D e M} \sqrt{S_{M}(z)}\right)=0 .
\end{aligned}
$$

$\bar{p}_{D f k}, A_{k}$, and $B_{k}(k=0,1, \ldots, M)$ can be obtained by solving the simultaneous equations (54)-(59). In (54)-(59), the tree-shaped fractal network parameters can be directly used to express the parameters of the permeability ratio, $k_{f(k+1)} / k_{f k}$, the dimensionless effective interface radius, $r_{D e k}$, and the function, $S_{k}(z)$.

The dimensionless effective interface radius expression can be derived by substituting (7) into (29):

$$
r_{\text {Dek }}=\frac{l_{0}}{r_{w}}\left[1+\frac{\alpha\left(1-\alpha^{k}\right) \cos \theta}{1-\alpha}\right] e^{S} .
$$

The permeability ratio can be calculated using (10):

$$
\frac{K_{f(k+1)}}{K_{f k}}= \begin{cases}n \beta^{2} \cos \theta, & k=0, \\ n \beta^{2}, & k>0 .\end{cases}
$$

The expression of the function $S_{k}(z)$ is as follows:

$$
S_{k}(z)=\frac{\left(K_{f 0} / K_{f k}\right) \lambda_{k}\left(1-\omega_{k}\right) z}{\left(K_{f 0} / K_{f k}\right)\left(1-\omega_{k}\right) e^{2 S} z+\lambda_{k} C_{D e}}+\frac{K_{f 0}}{K_{f k}} \frac{\omega_{k}}{C_{D e}} z,
$$

where

$$
\frac{K_{f 0}}{K_{f k}}=\left(n \beta^{2}\right)^{-k}
$$

The interporosity flow coefficient, $\lambda_{k}$, can be obtained by substituting (4) and (10) into (33), and the fluid capacitance coefficient, $\omega_{k}$, can be obtained by substituting (3), (14), and (15) into (32). 


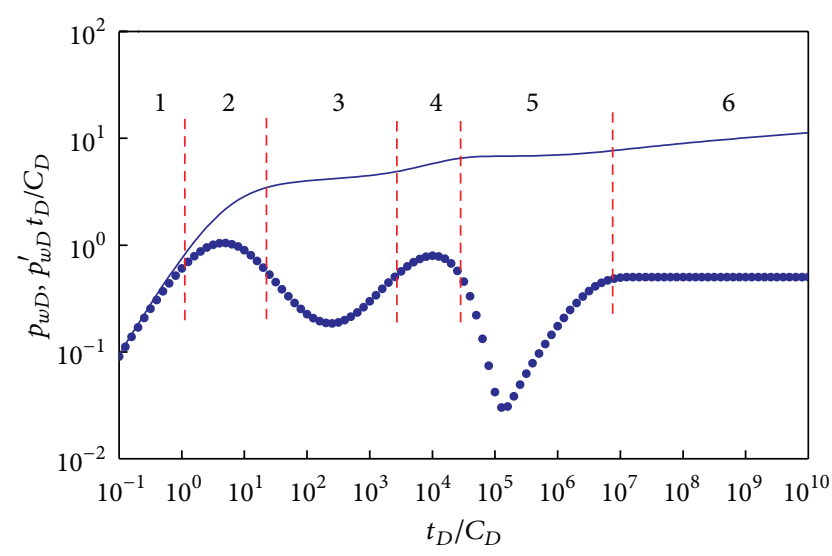

FIgURe 2: Pressure type curves of dual fractal reservoirs $(\alpha=1$, $\beta=0.707, \theta=1, N=4, M=10, l_{0}=10 \mathrm{~m}, d_{0}=0.05 \mathrm{~m}, D_{f}=1.5$, $T_{m}=4, r_{w}=0.1 \mathrm{~m}, S=-1, C_{D e}=0.0001, h=10 \mathrm{~m}, C_{m t}=$ $2.2 \times 10^{-5} \mathrm{MPa}^{-1}$, and $\left.C_{f t}=1 \times 10^{-4} \mathrm{MPa}^{-1}\right)$.

\section{Analysis of Type Curve Characteristics}

Dimensionless bottom hole pressure in Laplace space, $p_{w f D}$, is obtained by solving the linear equations (54)-(57) using the Stehfest numerical inversion method. The bilogarithmic type curves of the dual fractal reservoirs can then be illustrated.

In a condition of closed top and bottom boundary, the transient flow process, which has six flow regimes, can be clearly shown (Figure 2). The full and dashed lines represent pressure and pressure derivative curves, respectively. Regime 1 is the pure wellbore storage regime. Pressure and its derivative curves appear as upward straight lines with a slope of 1 . Regime 2 is the transition flow regime. The shape of the derivative curve looks like an "arch," which is influenced by the wellbore storage coefficient and skin factor. Regime 3 is the fracture system inter-porosity flow regime. The pressure derivative curve is $\mathrm{V}$ shaped, which depicts the response of inter-porosity flow between the fractures that are heterogeneously distributed. This inter-porosity flow regime is caused by spread of the pressure wave through the fracture system. Regime 4 is the fracture system radial flow regime. Slope of the pressure derivative curve is zero. In this scenario, the pressure wave spreads through the whole fracture system and begins to spread to the matrix system. Regime 5 is the inter-porosity flow regime of matrix system to fracture system. The pressure derivative curve is also $\mathrm{V}$ shaped. However, it is influenced by spread of the pressure wave through the matrix system. Regime 6 is the total system radial flow regime. The pressure derivative curve converges to a horizontal line, which depicts the response of the pressure dynamic balance state in the whole system.

Figure 3 shows the type curve characteristics affected by pore tortuosity, $T_{m}$. As $T_{m}$ increases, the two $\mathrm{V}$ shaped curves deepen and shift to the right, which indicates a longer lasting inter-porosity flow regime and a delayed occurring time of the radial flow regime in the fracture system. It also indicates a delayed inter-porosity flow regime from matrix to fracture. An increase in the initial branch number, $N$, has a similar

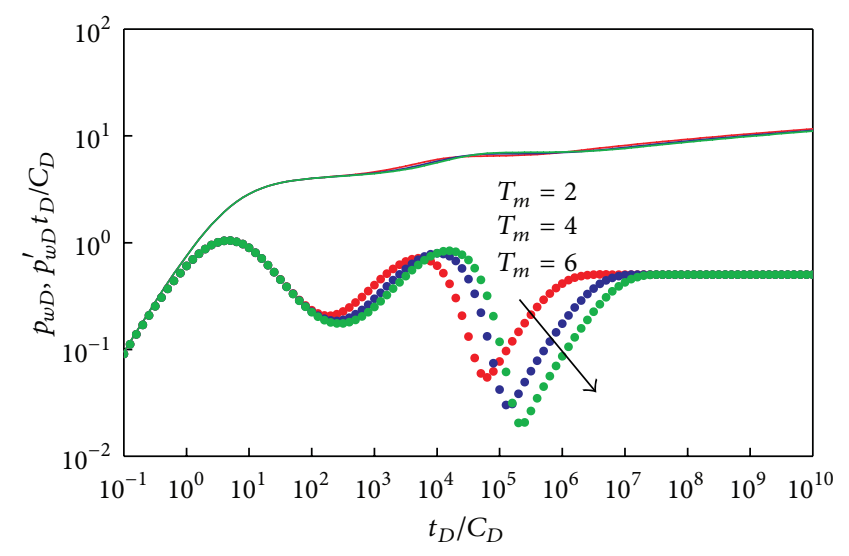

FIGURE 3: Effect of pore tortuosity $\left(T_{m}\right)$ on type curves $(\alpha=1, \beta=$ 0.707, $\theta=1, N=4, M=10, l_{0}=10 \mathrm{~m}, d_{0}=0.05 \mathrm{~m}, D_{f}=1.5$, $r_{w}=0.1 \mathrm{~m}, S=-1, C_{D e}=0.0001, h=10 \mathrm{~m}, C_{m t}=2.2 \times 10^{-5} \mathrm{MPa}^{-1}$, and $C_{f t}=1 \times 10^{-4} \mathrm{MPa}^{-1}$ ).

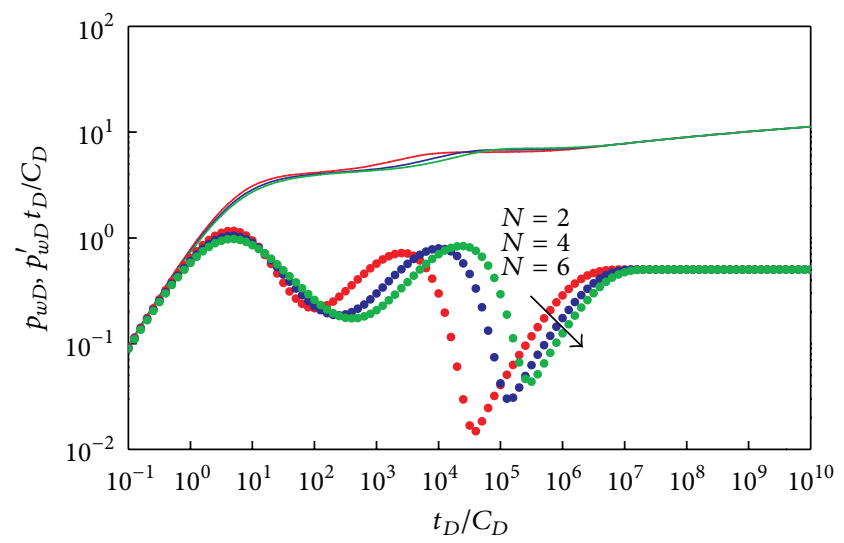

FIGURE 4: Effect of initial branch number $(N)$ on type curves $(\alpha=1$, $\beta=0.707, \theta=1, M=10, l_{0}=10 \mathrm{~m}, d_{0}=0.05 \mathrm{~m}, D_{f}=1.5, T_{m}=4$, $r_{w}=0.1 \mathrm{~m}, S=-1, C_{D e}=0.0001, h=10 \mathrm{~m}, C_{m t}=2.2 \times 10^{-5} \mathrm{MPa}^{-1}$, and $\left.C_{f t}=1 \times 10^{-4} \mathrm{MPa}^{-1}\right)$.

influence on flow regimes 3,4 , and 5 as an increase in $T_{m}$, but the $\mathrm{V}$ shaped curves associated with the pressure derivative become shallower and shift to the right (Figure 4).

Figure 5 exhibits the type curve characteristics affected by pore fractal dimension, $D_{f}$. Permeability of matrix system, $K_{m}$, and porosity of matrix system, $\phi_{m}$, increase with an increase in $D_{f}$. A larger $K_{m}$ leads to greater flow capacity in the matrix system with an earlier transition to regime 5. A larger $\phi_{m}$ leads to a larger supplying capacity in the matrix system, and regime 5 occurs earlier and lasts longer. As $D_{f}$ increases, regime 5 occurs earlier and lasts longer, which is depicted as a deeper and wider second $\mathrm{V}$ shaped pressure derivative curve.

Figure 6 shows the type curve characteristics affected by branch angle, $\theta$. Permeability of fracture system, $K_{f}$, decreases with an increase in $\theta$. A smaller $K_{f}$ leads to lower flow capacity in the fracture system with an earlier transition to regime 5 . Porosity of fracture system, $\phi_{f}$, increases with an 


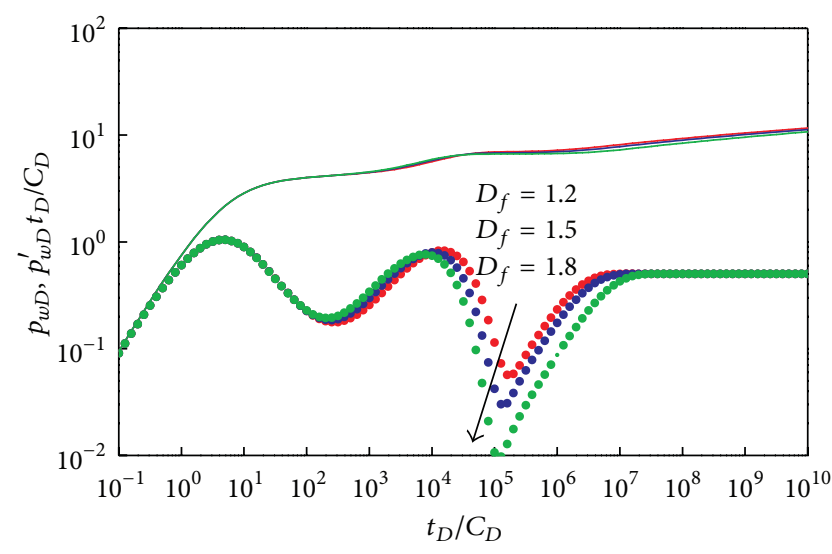

FigURE 5: Effect of pore fractal dimension $\left(D_{f}\right)$ on type curves $(\alpha=$ $1, \beta=0.707, \theta=1, N=4, M=10, l_{0}=10 \mathrm{~m}, d_{0}=0.05 \mathrm{~m}, T_{m}=4$, $r_{w}=0.1 \mathrm{~m}, S=-1, C_{D e}=0.0001, h=10 \mathrm{~m}, C_{m t}=2.2 \times 10^{-5} \mathrm{MPa}^{-1}$, and $C_{f t}=1 \times 10^{-4} \mathrm{MPa}^{-1}$ ).

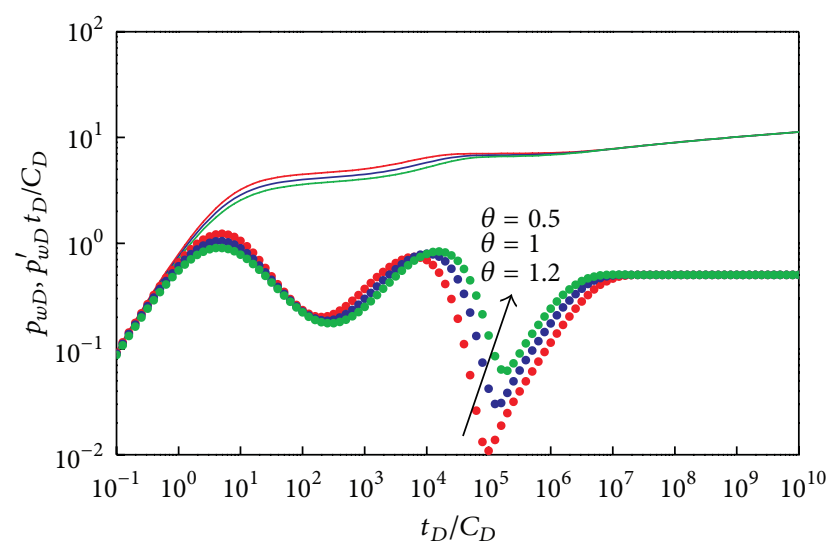

Figure 6: Effect of branch angle $(\theta)$ on type curves $(\alpha=1, \beta=$ 0.707, $N=4, M=10, l_{0}=10 \mathrm{~m}, d_{0}=0.05 \mathrm{~m}, D_{f}=1.5, T_{m}=4$, $r_{w}=0.1 \mathrm{~m}, S=-1, C_{D e}=0.0001, h=10 \mathrm{~m}, C_{m t}=2.2 \times 10^{-5} \mathrm{MPa}^{-1}$, and $C_{f t}=1 \times 10^{-4} \mathrm{MPa}^{-1}$ ).

increase in $\theta$. A larger $\phi_{f}$ leads to greater supplying capacity in the fracture system, and regime 5 occurs later with a shorter duration. When $\theta$ increases, it has the opposite effect on flow regime 5 as an increase in $D_{f}$.

Figure 7 exhibits the type curve characteristics affected by the length ratio, $\alpha . \phi_{f}$ decreases with an increase in $\alpha$. A smaller $\phi_{f}$ leads to a lower supplying capacity in the fracture system and an earlier transition to a longer lasting regime 5 . When a large $\alpha$ increases, regime 5 occurs earlier, which manifests in a deeper and wider second $\mathrm{V}$ shaped type curve.

Figure 8 exhibits the type curve characteristics affected by diameter ratio, $\beta . K_{f}$ and $\phi_{f}$ increase with an increase in $\beta$. A larger $K_{f}$ leads to larger flow capacity in the fracture system and a later transition to regime 5 . A larger $\phi_{f}$ leads to greater supplying capacity in the fracture system with a later and shorter regime 5 . When $\beta$ is smaller than $0.707, K_{f}$ increases with an increase in $r$, and when $\beta$ is greater than $0.707, K_{f}$ decreases with $r$. $\beta$ affects all regimes except for

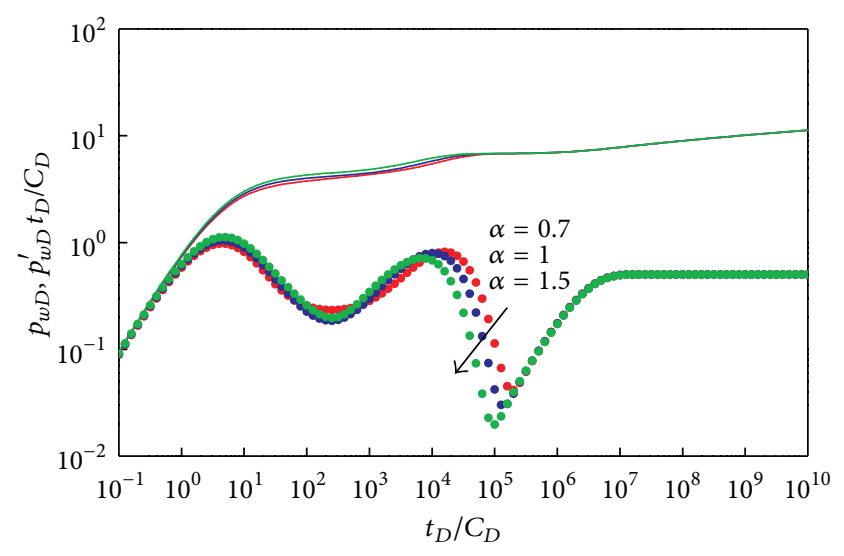

Figure 7: Effect of length ratio $(\alpha)$ on type curves $(\beta=0.707, \theta=1$, $N=4, M=10, l_{0}=10 \mathrm{~m}, d_{0}=0.05 \mathrm{~m}, D_{f}=1.5, T_{m}=4, r_{w}=$ $0.1 \mathrm{~m}, S=-1, C_{D e}=0.0001, h=10 \mathrm{~m}, C_{m t}=2.2 \times 10^{-5} \mathrm{MPa}^{-1}$, and $\left.C_{f t}=1 \times 10^{-4} \mathrm{MPa}^{-1}\right)$.

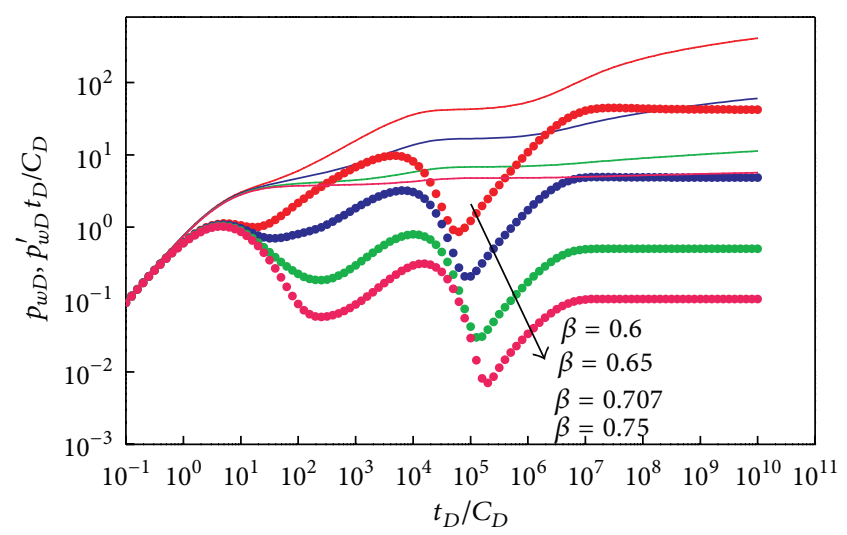

Figure 8: Effect of diameter ratio $(\beta)$ on type curves $(\alpha=1, \theta=1$, $N=4, M=10, l_{0}=10 \mathrm{~m}, d_{0}=0.05 \mathrm{~m}, D_{f}=1.5, T_{m}=4, r_{w}=$ $0.1 \mathrm{~m}, S=-1, C_{D e}=0.0001, h=10 \mathrm{~m}, C_{m t}=2.2 \times 10^{-5} \mathrm{MPa}^{-1}$, and $\left.C_{f t}=1 \times 10^{-4} \mathrm{MPa}^{-1}\right)$.

pure wellbore storage and transition flow regime, which are not affected by $K_{f}$. A larger $\beta$ leads to a lower location of the dimensionless pressure curve, and regime 5 occurs later, resulting in a shallower and narrower second $\mathrm{V}$ shaped type curve. When $\beta$ equals the critical value of 0.707 (16), the horizontal line representing regime 6 equals 0.5 .

Figures 9 and 10 exhibit the type curve characteristics affected by total branch level, $M$, when diameter ratio, $\beta$, is 0.65 and 0.75 , respectively. Radius, $r$, increases with an increase in $M$. Additionally, $r$ enhances the type curve characteristics affected by $M$. When $\beta$ is less than 0.707 , a large $M$ leads to a higher dimensionless pressure curve, and, vice versa, when $\beta$ is greater than 0.707 , a large $M$ leads to a lower dimensionless pressure curve.

\section{Conclusions}

The transient flow model for pressure responses in dual fractal reservoirs is established and solved, type curves are 


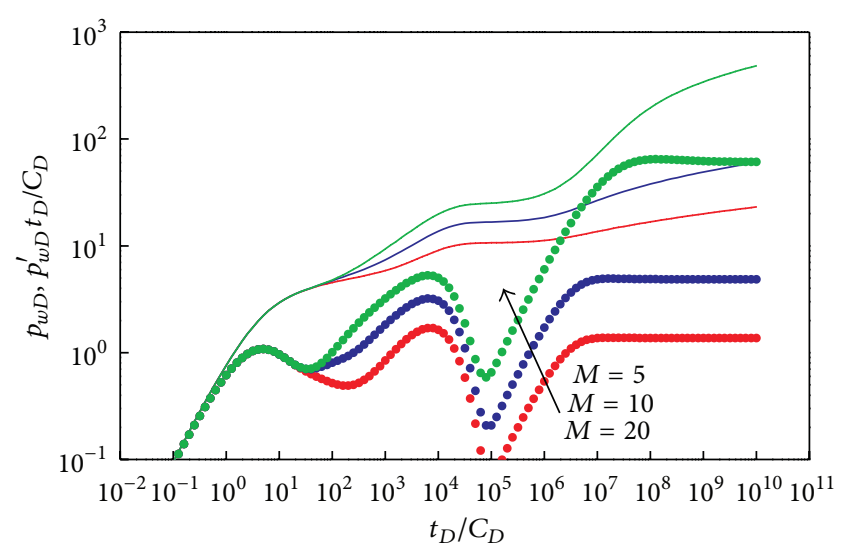

FIGURE 9: Effect of total branch level $(M)$ on type curves $(\alpha=1$, $\beta=0.65, \theta=1, N=4, M=10, l_{0}=10 \mathrm{~m}, d_{0}=0.05 \mathrm{~m}, D_{f}=1.5$, $T_{m}=4, r_{w}=0.1 \mathrm{~m}, S=-1, C_{D e}=0.0001, h=10 \mathrm{~m}, C_{m t}=$ $2.2 \times 10^{-5} \mathrm{MPa}^{-1}$, and $\left.C_{f t}=1 \times 10^{-4} \mathrm{MPa}^{-1}\right)$.

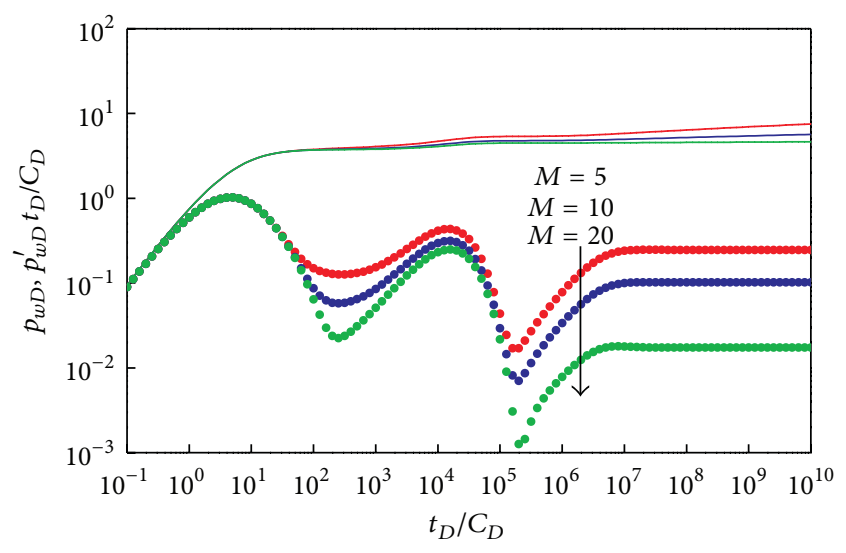

FIGURE 10: Effect of total branch level $(M)$ on type curves $(\alpha=1$, $\beta=0.65, \theta=1, N=4, M=10, l_{0}=10 \mathrm{~m}, d_{0}=0.05 \mathrm{~m}, D_{f}=1.5$, $T_{m}=4, r_{w}=0.1 \mathrm{~m}, S=-1, C_{D e}=0.0001, h=10 \mathrm{~m}, C_{m t}=$ $2.2 \times 10^{-5} \mathrm{MPa}^{-1}$, and $\left.C_{f t}=1 \times 10^{-4} \mathrm{MPa}^{-1}\right)$.

illustrated, and dual fractal flow behavior characteristics are analyzed. The following conclusions were obtained.

(1) Fracture and matrix systems can be simulated using a tree-shaped fractal network and fractal porous media, respectively.

(2) Six flow regimes for pressure type curves can be established. Type curves are dominated by fracture and matrix fractal parameters. These various parameters affect type curves differently.

(3) Type curves are dominated by external boundary conditions, fractal parameters the fluid capacitance coefficient and the inter-porosity flow factor.

(4) Semianalytical dual fractal modeling is suitable for various naturally fractured oil or gas reservoirs and provides a practical method to solve empirical cases.

\section{Acknowledgments}

The authors are grateful for financial support from the National Science Fund for Distinguished Young Scholars of China (Grant no. 51125019), the National Key Basic Research and Development Program of China (Grant no. 2011CB201005), and the Science and Technology Innovation Fund of Southwest Petroleum University (Grant no. GIFSB0701).

\section{References}

[1] R. Camacho-Velázquez, G. Fuentes-Cruz, and M. VásquezCruz, "Decline-curve analysis of fractured reservoirs with fractal geometry," SPE Reservoir Evaluation and Engineering, vol. 11, no. 3, pp. 606-619, 2008.

[2] Y. G. Zhang and D. K. Tong, "The pressure transient analysis of deformation of fractal medium," Journal of Hydrodynamics, vol. 20, no. 3, pp. 306-313, 2008.

[3] A. Jafari and T. Babadagli, "Generating 3D permeability map of fracture networks using well, outcrop, and pressure-transient data," SPE Reservoir Evaluation and Engineering, vol. 14, no. 2, pp. 215-224, 2011.

[4] L. Zhang, J. Zhang, and Y. Zhao, "Analysis of a finite element numerical solution for a nonlinear seepage flow model in a deformable dual media fractal reservoir," Journal of Petroleum Science and Engineering, vol. 76, no. 3-4, pp. 77-84, 2011.

[5] W. Wechsatol, S. Lorente, and A. Bejan, "Tree-shaped insulated designs for the uniform distribution of hot water over an area," International Journal of Heat and Mass Transfer, vol. 44, no. 16, pp. 3111-3123, 2001.

[6] W. Wechsatol, S. Lorente, and A. Bejan, "Optimal tree-shaped networks for fluid flow in a disc-shaped body," International Journal of Heat and Mass Transfer, vol. 45, no. 25, pp. 4911-4924, 2002.

[7] P. $\mathrm{Xu}$ and B. Yu, "The scaling laws of transport properties for fractal-like tree networks," Journal of Applied Physics, vol. 100, no. 10, Article ID 104906, 8 pages, 2006.

[8] P. Xu, B. Yu, Y. Feng, and Y. Liu, "Analysis of permeability for the fractal-like tree network by parallel and series models," Physica A, vol. 369, no. 2, pp. 884-894, 2006.

[9] P. Xu, B. Yu, M. Yun, and M. Zou, "Heat conduction in fractal tree-like branched networks," International Journal of Heat and Mass Transfer, vol. 49, no. 19-20, pp. 3746-3751, 2006.

[10] M. Yun, B. Yu, and J. Cai, "A fractal model for the starting pressure gradient for Bingham fluids in porous media," International Journal of Heat and Mass Transfer, vol. 51, no. 5-6, pp. 1402-1408, 2008.

[11] S. Wang, B. Yu, Q. Zheng, Y. Duan, and Q. Fang, "A fractal model for the starting pressure gradient for Bingham fluids in porous media embedded with randomly distributed fractal-like tree networks," Advances in Water Resources, vol. 34, no. 12, pp. 1574-1580, 2011.

[12] B. Yu and P. Cheng, "A fractal permeability model for bidispersed porous media," International Journal of Heat and Mass Transfer, vol. 45, no. 14, pp. 2983-2993, 2002.

[13] J. Bear, Dynamics of Fluids in Porous Media, American Elsevier, 1972.

[14] M. Yun, B. Yu, and J. Cai, "Analysis of seepage characters in fractal porous media," International Journal of Heat and Mass Transfer, vol. 52, no. 13-14, pp. 3272-3278, 2009. 
[15] J. E. Warren and P. J. Root, "The behavior of naturally fractured reservoirs," Society of Petroleum Engineers Journal, vol. 3, no. 3, pp. 245-255, 1963.

[16] R. S. Nie, J. C. Guo, Y. L. Jia, S. Q. Zhu, Z. Rao, and C. G. Zhang, "New modelling of transient well test and rate decline analysis for a horizontal well in a multiple-zone reservoir," Journal of Geophysics and Engineering, vol. 8, no. 3, article 464, 2011.

[17] R. G. Agarwal, R. Al-Hussainy, and H. J. Ramey Jr., "Investigation of wellbore storage and skin effect in unsteady liquid flow1," Society of Petroleum Engineers Journal, vol. 10, no. 3, pp. 279290, 1970.

[18] D. Bourdet and A. C. Gringarten, "Determination of fissure volume and block size in fractured reservoirs by type-curve analysis," in Proceedings of the SPE Annual Technical Conference and Exhibition, American Institute of Mining, Metallurgical and Petroleum Engineers, Dallas, Tex, USA, September 1980.

[19] R. C. Earlougher, Advances in Well Test Analysis, Henry L. Doherty Memorial Fund of AIME, New York, NY, USA, 1977.

[20] A. F. V. Everdingen and W. Hurst, "The application of the laplace transformation to flow problems in reservoirs," Journal of Petroleum Technology, vol. 1, no. 12, pp. 305-324, 1949. 


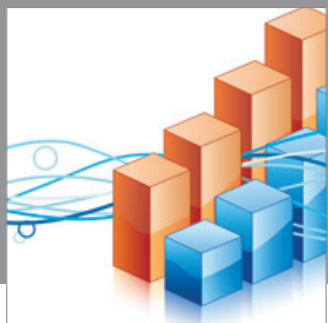

Advances in

Operations Research

mansans

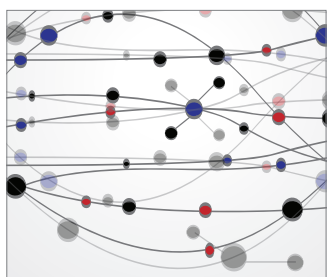

The Scientific World Journal
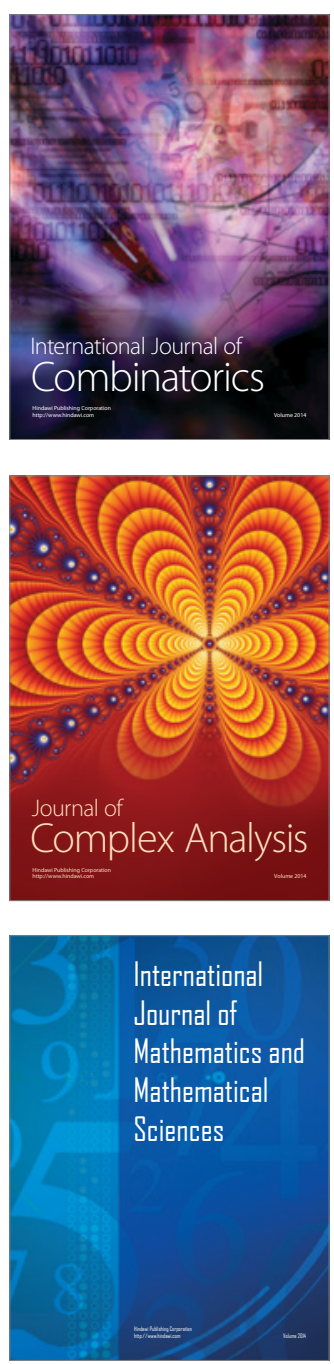
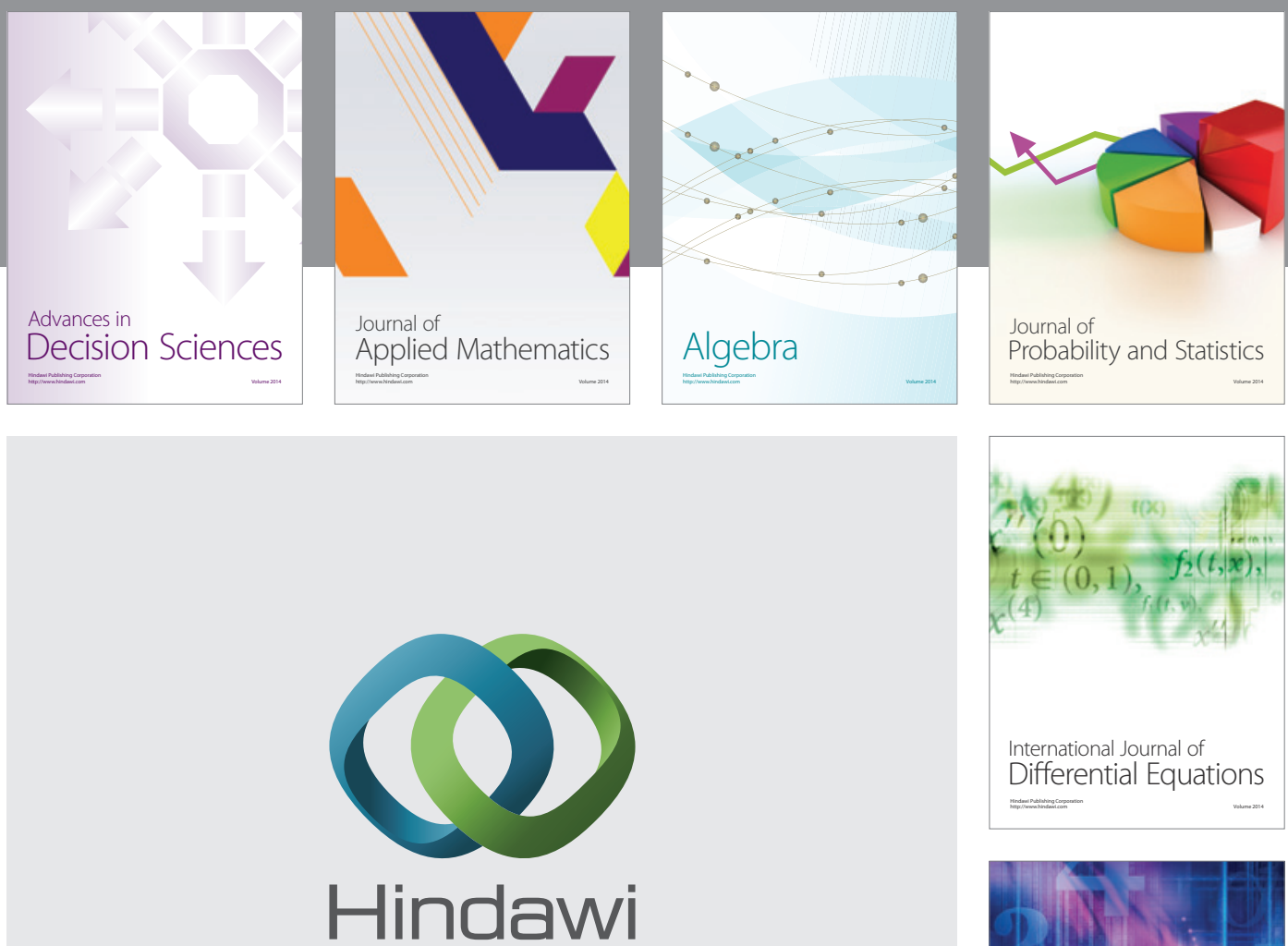

Submit your manuscripts at http://www.hindawi.com
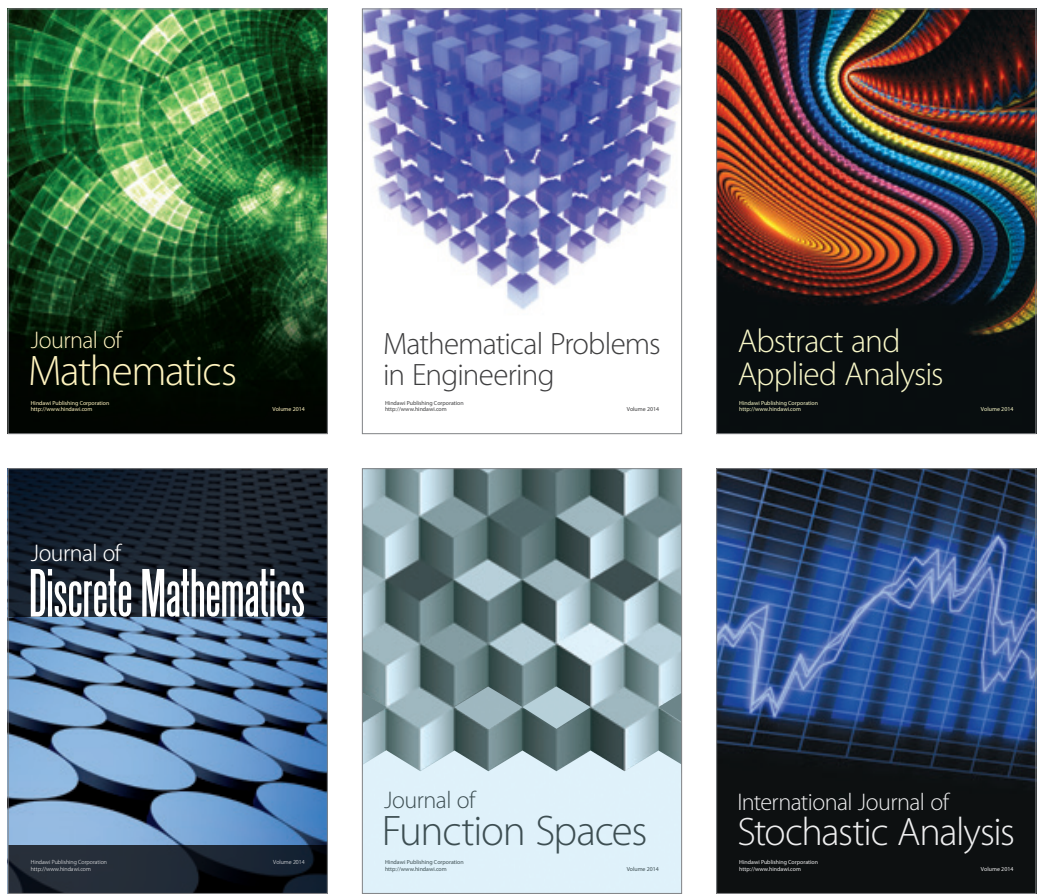

Journal of

Function Spaces

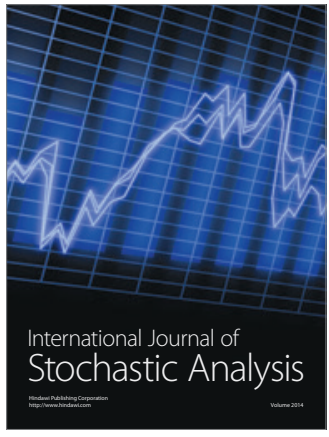

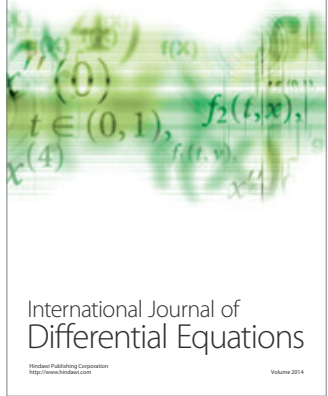
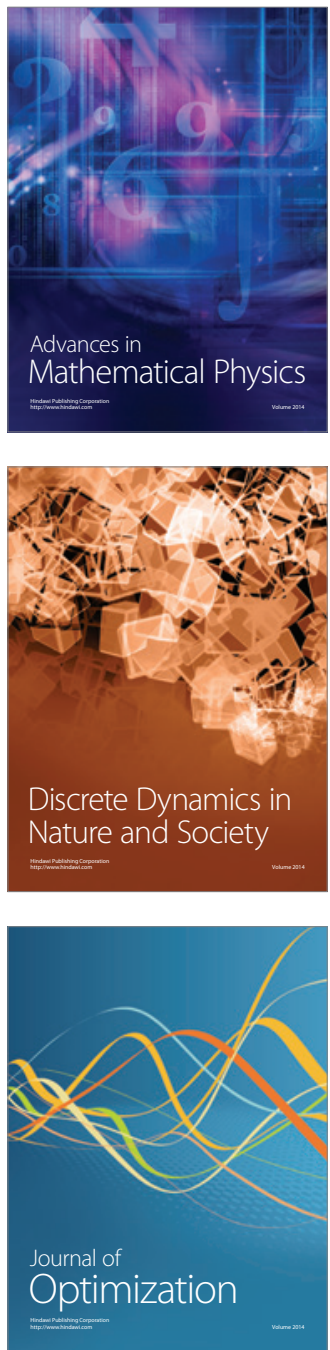